FORMATION Formation emploi

Revue française de sciences sociales

116 | octobre-décembre 2011

Pêle-mêle

\title{
La relation formation/carrières artistiques : le paradoxe des mondes de l'art
}

The relationship of training to careers in the arts: the paradox of the art worlds

Die Beziehung Ausbildung/künstlerische Laufbahn: das Paradoxon der

Kunstwelten

La relación formación/carreras artísticas : la paradoja de los mundos del arte

Nathalie Moureau et Dominique Sagot-Duvauroux

\section{OpenEdition}

Journals

Édition électronique

URL : http://journals.openedition.org/formationemploi/3463

DOI : 10.4000/formationemploi.3463

ISSN : 2107-0946

Éditeur

La Documentation française

Édition imprimée

Date de publication : 30 décembre 2011

Pagination : $35-49$

ISSN : 0759-6340

Référence électronique

Nathalie Moureau et Dominique Sagot-Duvauroux, «La relation formation/carrières artistiques : le paradoxe des mondes de l'art », Formation emploi [En ligne], 116 | octobre-décembre 2011, mis en ligne le 24 janvier 2012, consulté le 30 octobre 2020. URL : http://journals.openedition.org/ formationemploi/3463 ; DOI : https://doi.org/10.4000/formationemploi.3463 


\title{
Formation \\ Profession
}

\section{La relation formation/carrières artistiques : le paradoxe des mondes de l'art'}

Nathalie Moureau et Dominique Sagot-Duvauroux

\begin{abstract}
Cet article éclaire les liens parodoxaux qui existent entre les modes d'apprentissage du métier d'artiste et les différents mondes de l'art.
\end{abstract}

Quel rôle joue la formation dans les carrières artistiques? Les arts plastiques constituent un domaine où la place de la formation pose encore question; et ce, bien que de nombreuses recherches économiques aient été conduites sur le marché du travail artistique (pour une revue de la littérature, voir Alper Wassall, 2006 ; Benhamou, 2004 ; Menger, 2006 ; 2009 ; Towse, 2006). Les liens complexes qui existent entre formation, capital humain et rémunération, qui ont largement été discutés dans la littérature sur l'éducation (Becker, 1964 ; Arrow, 1973 ; Spence, 1973 ; Mingat et Eicher, 1975, etc.), sont rendus encore plus opaques par la nécessaire introduction de la variable « talent ». Commentant les écrits de Rosen (1987), qui considère qu'en moyenne seulement un tiers des diffé-

\footnotetext{
${ }^{1}$ Cet article utilise une partie des résultats d'un contrat de recherche effectué pour le compte du ministère de la Culture sur la valorisation et la diffusion de l'art contemporain en région ; cinq agglomérations ont été étudiées : Le Havre, Lyon, Montpellier, Nantes et Rouen. Cinq chercheurs ont participé à ce contrat : Muriel De Vrièse, Bénédicte Martin, Corinne Melin Nathalie Moureau et Dominique Sagot Duvauroux. L'analyse en composante principale, réalisée sur les données collectées, ainsi que la grille d'entretien qui a été utilisée pour conduire les entretiens auprès des artistes sont présentées dans la suite de ce document.
}

Nathalie Moureau est maître de conférence en sciences économiques, université de Montpellier 3, membre du LAMETA (LAboratoire Montpelliérain d'Economie Théorique et Appliquée) (UMR 5272 CNRS université Montpellier I Supagro Inra). Elle est spécialisée en économie de la culture. Elle a publié récemment : Clément V., Moureau N., Vidal M. (2009), " À la recherche des biens sous tutelle», L'Actualité Economique, 85 (4). Moureau N., Vidal M. (2009), "Quand préférences déclarées et révélées s'opposent: Le téléspectateur, un cas paradoxal pour l'économiste ", Revue Française de Sociologie Economique, 4, pp. 199-218. Benhamou F., Gergaud O., Moureau N. (2009), "Les stratégies de différenciation des produits par la télévision. Une analyse économétrique des caractéristiques des films financés par les chaînes ", Economie et Prévision, 188 (2), pp. 101-112.

Dominique Sagot-Duvauroux est professeur des Universités, directeur du GRANEM UMR-MA IGroupe de Recherche ANgevin en Economie et Management, Unité Mixte de Recherche-ministère de l'Agriculture, $n^{\circ 0} 49$ |, spécialiste d'économie de la culture léconomie des droits de propriété intellectuelle, 
marché de l'art, photographie, intervention publique, culture et numérique). II a publié récemment : Le marché de l'art contemporain, "Repères 》, La Découverte, $2006 / 2010$ en coll. Avec N. Moureau, "Economies des droits d'auteur : V-Synthèse », Culture Etudes, ministère de la Culture, la Documentation française, 2007-8, décembre 2007, 16 p., en coll. avec F. Benhamou. http://www2.culture.gouv.fr/deps/pdf/etudes/ CE_2007-8_synthese_BAT051207.pdf. " Mutation des filières, recomposition des modèles économiques et partage des risques économiques », in F. Labadie, F. Rouet, Travail artistique et économie de la création, collection "Questions de Culture », la Documentation française, 2008, pp. 39-54.

rences de rémunérations sont imputables à des différences d'investissement en capital humain, le reste résultant d'effets individuels et d'événements aléatoires, Towse (2006) souligne combien ces derniers effets sont importants pour les artistes en raison du poids prégnant des variations de talent et de chance dans leur profession (Towse, p. 873). L'importance du talent rendrait-elle inutile le rôle de l'école?

Nombreux sont les auteurs qui soulignent l'importance du talent dans les carrières artistiques ; cependant, peu se hasardent à en donner une définition. Dans le modèle de superstar qu'il a développé, Rosen (1981) montre comment de petites différences de talent peuvent s'accompagner de grandes variations de revenus. Il évite cependant la question de sa définition et de sa mesure et indique "qu'une mesure cardinale de la qualité ou du talent repose sur la mesure des revenus » (Rosen, 1981, p. 848). Comme le souligne Menger (2010), chez Rosen, « le talent est une variable exogène dont le calibrage est incertain». Quelques économistes se sont toutefois attachés à donner une définition économique du talent. Ruth Towse (2006) le définit en s'appuyant sur la théorie ricardienne de la rente : il s'agit d'un don de la nature, d'un capital inné. Celui qui en est doté acquiert plus aisément un savoir faire et peut également atteindre un haut niveau de productivité artistique - hautes performances - avec lesquelles peu de concurrents peuvent rivaliser.

Le talent, bien qu'inné, ne semble donc pas remettre totalement en cause le rôle de la formation. Celle-ci peut permettre de le révéler à travers un mécanisme de sélection adapté ou de le développer grâce à l'apprentissage de connaissances qui vont se greffer sur lui et permettre son expression. Outre des savoirfaire, l'école contribue aussi à socialiser l'artiste, à l'insérer dans des réseaux et à lui apprendre comment se comporter dans le monde professionnel (Towse, 200 ; 2006, Menger, 2009 ; Caves, 2000). Pour Blaug (1985), dans les activités artistiques, les trois S, i.e. skilling, screening et socialisation, sont aussi importants pour la formation que les trois $\mathrm{Rs}^{2}$, i.e. Reading, wRiting and aRithmetic pour les secteurs traditionnels. En effet, acquérir des compétences, évaluer ses capacités mais aussi obtenir des recommandations de professionnels reconnus ou encore s'insérer dans les réseaux contribuent à favoriser la carrière des artistes.

La plupart de ces travaux ont cependant tendance à considérer l'art et les artistes comme des catégories relativement homogènes. Les travaux conduits par Howard Becker (1988), en sociologie de l'art, ont montré qu'il n'en était rien ; coexistaient en effet différents mondes de l'art, régis par des conventions de qualité distinctes ${ }^{3}$ et des intermédiaires spécifiques. Par la suite, le rôle joué par les réseaux et les conventions artistiques a été exploré, par exemple dans le cadre des musicos (Perrenoud, 2008), des scénaristes (Bielby D., 2009), ou encore pour analyser les différences de carrières entre hommes et femmes (Buscatto M., 2007). S'agissant du domaine qui nous intéresse ici, les arts plastiques, deux spécialités distinctes coexistent selon Caves (2000), l'une fondée sur le savoir-faire, l'autre sur les idées créatives et la recherche (p. 22). L'existence de différentes conventions de qualité sur le marché de l'art contemporain a largement été approfondie (Heinich, 1999 ;

2 Les trois Rs correspondent aux enseignements fondamentaux du système éducatif américain : Reading, wRiting and aRithmetic. Cette séquence est attribuée à Sir William-Curtis ((autour de 1825). Depuis, le mythe des trois Rs a persisté bien qu'il ait renvoyé depuis à d'autres modèles (e.g. Read, wRite, Recite).

3 La coordination sur le marché ne s'effectue pas ex nihilo mais suppose l'existence d'un monde commun, d'un système de représentation partagé selon les individus. Pour être efficaces, les comportements doivent mobiliser une forme de savoir qui porte sur le collectif et qu'on appelle ici une convention. Les conventions esthétiques sur le marché de l'art fournissent aux agents les procédures qui leur permettent de distinguer les œuvres de qualité des mauvaises. Elles identifient les caractéristiques à prendre en compte pour juger de la qualité et associent à ces caractéristiques des échelles de valeur. Elles permettent donc une classification ordinale des oeuvres et une différenciation verticale du marché. 
Moureau Sagot-Duvauroux, 1992 ; 2010, Martin, 2005). À chacune de ces conventions correspondent des systèmes d'évaluation différents qui s'inscrivent dans la typologie proposée par Wijnberg (2000) : le marché, les pairs, les experts, chacun des systèmes étant défini en fonction d'un processus concurrentiel particulier i.e. la façon dont les vainqueurs sont distingués des perdants. Avec le marché, les sélectionneurs sont les acheteurs et les producteurs sont les sélectionnés. Avec les pairs, les sélectionneurs et sélectionnés appartiennent au même groupe ; enfin, avec les experts, les sélectionneurs appartiennent à un groupe particulier et tirent leur pouvoir de sélection de leur savoir spécialisé.

Dès lors que les critères de qualité diffèrent d'un monde à l'autre, on peut supposer que les mécanismes d'apprentissage nécessaires pour exceller dans chaque groupe sont eux aussi distincts. Le but de cet article est d'explorer le lien qui existe entre les choix esthétiques auxquels un artiste adhère et le type de formation qu'il a suivi plus que d'expliquer les différences de carrière des artistes selon leur sexe, leur âge ou leurs origines sociales.

Nous chercherons ainsi à éclairer un paradoxe relatif à la relation formation/carrières artistiques. Alors que l'histoire de l'art, depuis le début du XXe siècle, s'est employée à dissocier le statut d'artiste de la maîtrise d'un savoir faire plastique (dessin, peinture, sculpture, etc.) en valorisant avant tout l'originalité de la démarche, les écoles des beaux-arts apparaissent encore aujourd'hui comme un passage quasi incontournable pour une carrière artistique sur le marché de l'art contemporain. Inversement, alors que la maîtrise d'un savoir faire artisanal apparaît comme le principal critère d'appréciation de la qualité des artistes dans le monde de l'art classique s'inscrivant dans la tradition, il n'est pas rare que ces artistes soient autodidactes. On sait qu'historiquement, le glissement progressif de l'artiste artisan (corporations) vers l'artiste savant (académie) et enfin vers l'artiste inspiré s'est accompagné d'une évolution des dispositifs d'apprentissage (atelier de maître, écoles des beaux-arts, autodidactie) (Heinich, 1993).

Reprenant le concept de monde de l'art de Becker (1988), nous identifierons, dans un premier temps, différents mondes de l'art et les types d'artistes qui leur sont associés. Dans un second temps, partant de cette typologie, nous analyserons les raisons du rôle différencié que joue la formation dans les différents mondes. Nous nous appuierons sur les résultats d'une enquête conduite pour le ministère de la Culture sur la valorisation et la diffusion de l'art contemporain dans plusieurs agglomérations françaises : Le Havre, Rouen, Lyon, Nantes et Montpellier. À cet effet, des entretiens ont été conduits auprès de 203 acteurs de l'art actuel, dont 69 artistes, dont nous avons retranscrit le parcours.

\section{MONDES DE L'ART ET PROFILS D'ARTISTES}

De l'enquête conduite auprès des acteurs de l'art en région, il ressort une opposition nette entre un monde de l'art « classique $»^{4}$, mettant en avant le savoir-faire « artisanal » des artistes et leur capacité d'émouvoir, et un monde de l'art « contemporain », au sein duquel l'innovation et la recherche sont les principaux attributs du talent. Ce dernier s'organise autour d'institutions clés comme le Fonds régional d'art contemporain (FRAC), les écoles des beaux-arts et les centres d'art contemporain labellisés, et fonctionne en réseau très dense. Le monde de l'art classique, à l'opposé, apparaît isolé, à la fois par rapport aux institutions et par rapport aux autres acteurs. Il n'intègre pratiquement pas de réseaux locaux d'acteurs, sauf du côté des artistes qui souvent auto-organisent leur diffusion. Quelques acteurs se trouvent à la périphérie des deux

\footnotetext{
${ }^{4}$ Nous reprenons ici les termes utilisés par Nathalie Heinich (1999) pour qualifier ce qu'elle appelle les différents genres de l'art actuel. Elle en distingue trois au sein de l'art actuel : l'art classique, l'art moderne et l'art contemporain. Ces trois appellations ne renvoient pas à un découpage historique de l'art mais bien à des œuvres actuelles relevant de critères de jugements distincts. Tandis que l'art "classique" respecte les règles classiques de figuration, de perspectives et les canons esthétiques, l'art "moderne" rompt avec les règles de la figuration classique mais respecte l'usage des matériaux traditionnels et exige une intériorité de l'artiste en gage d'authenticité de la démarche. L'art contemporain se démarque nettement des précédents dans le mesure où il est fondé sur la transgression des frontières qui définissent l'art pour le sens commun ; il correspond au genre de l'art actuel qui est valorisé par les institutions et dont il est fait largement écho dans la presse au travers des prix records atteints aux enchères par quelques-uns de ses représentants.
} 


\section{Encadré 1 \\ Méthodologie}

L'enquête a été conduite auprès de 134 acteurs de l'art (*) et de 69 artistes dans les agglomérations de Lyon, Le Havre/Rouen, Montpellier et Nantes (cf. tableau 1 pour une présentation de l'échantillon), choisies dans le but d'obtenir un panorama des modes de valorisation et de diffusion, qu'ils soient marchands ou non, privés ou publics, associatifs ou institutionnels.

\section{Tableau 1. Caractéristiques de l'échantillon d'artistes}

\begin{tabular}{|l|c|c|}
\hline Caractéristiques des artistes & Nombre & $\%$ \\
\hline Agglomération & & \\
\hline Le Havre Roven & 18 & 26 \\
\hline Lyon & 18 & 26 \\
\hline Montpellier & 20 & 29 \\
\hline Nantes & 13 & 19 \\
\hline Genre & & \\
\hline Hommes & 57 & 83 \\
\hline Femmes & 11 & 16 \\
\hline Couple (1) & 1 & 1 \\
\hline Formation & & \\
\hline Beaux-arts & 44 & 64 \\
\hline Autres formations artistiques & 8 & 11 \\
\hline Autodidactes & 17 & 25 \\
\hline Âge & & \\
\hline - de 40 ans & 18 & 26 \\
\hline $40-60$ ans & 35 & 50 \\
\hline+60 ans & 16 & 24 \\
\hline Medium dominant & & \\
\hline Peinture, dessin, collage & 27 & 39 \\
\hline Sculpture & 8 & 11 \\
\hline Photographie & 5 & 7 \\
\hline Video, Instal., performances & 14 & 20 \\
\hline Plasticiens, divers & 15 & 22 \\
\hline Total & 69 & 100 \\
\hline
\end{tabular}

Note de lecture : Sur 69 artistes interrogés, 18 résidaient dans les agglomérations du Havre ou de Rouen, 18 dans l'agglomération de Lyon, 20 dans celle de Montpellier, et 13 dans l'agglomération Nantaise. Parmi ces artistes, on compte 57 hommes, 11 femmes et deux personnes travaillant en couple et interrogées de ce fait de façon simultanée. 44 ont reçu une formation à l'école des beaux-arts, 17 sont autodidactes et 8 ont reçu une formation artistique distincte de celle des Beaux arts.

Dans chaque agglomération, nous avons commencé par interroger les principaux acteurs de l'art (hors artistes), toutes tendances esthétiques confondues, sur leur activité, leurs stratégies et sur les relations avec les autres acteurs. Des matrices de relation ont été construites pour réaliser une analyse de réseau. Celle-ci met en évidence différents mondes de l'art auxquels sont associées différentes économies, distinguées selon leurs rapports à l'innovation (De Vriese et alii, 2011). Lors de cette première enquête, nous avons demandé aux diffuseurs de nous proposer des noms d'artistes dont les carrières semblaient caractéristiques de l'économie au sein de laquelle ils évoluaient. Nous avons retenu 69 noms répartis dans les différentes régions et les différents mondes.

Notre échantillon n'a donc pas vocation à être statistiquement représentatif de la structure de la population des artistes. Il inclut des représentants « emblématiques » des différentes économies de l'art identifiées, de façon à pouvoir tester la relation entre formation et mondes de l'art. Il n'est donc pas exploitable pour établir une relation statistiquement pertinente entre sexe, âge ou origine sociale par exemple et réussite professionnelle.

À partir d'entretiens semi-directifs visant à retracer le parcours des artistes interrogés, (nature de la formation, lieux d'exposition et de ventes, intermédiaires avec lesquels ils travaillent, mode de fixation du prix, rencontres décisives pour la carrière, relais institutionnels, etc.), nous avons construit un tableau de données avec en ligne les artistes et en ordonnée une série de variables qualitatives relatives à la

Source : Etude réalisée pour le DEPS, ministère de la Culture. Synthèse de l'étude disponible sur le site du ministère : Culture études 2011.1 formation suivie, à l'activité d'exposition et de vente, au rapport à la commande, à l'inscription territoriale de l'activité ou encore au
soutien des institutions. À partir de ce tableau, nous avons réalisé une analyse factorielle de correspondances multiples qui a permis de dégager des profils emblématiques d'artistes $(* *)$.

$\left(^{*}\right)$ marchands ou associatifs, institutionnels ou relevant de la tutelle des collectivités territoriales.

(**) De façon plus précise, la liste des variables retenues pour l'analyse des correspondances était la suivante : sexe, âge, medium utilisé (peinture, sculpture, vidéo/installation), formation (beaux arts, arts appliqués, autres écoles d'art, autodidacte), institutions (FRAC-Fonds régional d'art contemporain, DRAC-Direction régionale des Affaires culturelles) profession (professeur école d'art, professeur école d'art appliqué, autres, artiste uniquement) ; diffusion (galerie locale privée, galerie locale associative, galerie privée à l'international) réseaux (commande publique, services, association d'artistes, collectifs), expositions (salons, foires, expositions libres, internationales). 
mondes et renvoient à ce que Nathalie Heinich (1998) qualifie « d'art moderne ».

L'analyse factorielle réalisée à partir des entretiens d'artistes permet de retrouver cette opposition entre les mondes. Elle fait apparaître également un autre critère de différentiation qui est le rapport de l'artiste à la commande et à son territoire. Les deux premiers axes du schéma 1 dessinent ainsi quatre profils d'artistes.

L'axe horizontal distingue principalement les artistes selon le type d'art pratiqué : tandis que les cadrans sud sont ceux de la tradition (art classique), les cadrans nord correspondent à l'innovation (art contemporain). Les variables les plus discriminantes sur cet axe sont le medium utilisé (les termes peintre ou sculpteur étant généralement revendiqués par les artistes s'ancrant dans la tradition tandis que ceux de vidéaste, plasticien ou photographe renvoient davantage à l'art de recherche), la formation (les artistes autodidactes s'opposant aux artistes ayant suivi une école des beaux-arts), le soutien institutionnel (FRAC, DRAC) marquant très clairement l'art contemporain, la présence dans les salons caractérisant à l'opposé les artistes classiques.

L'axe vertical oppose les artistes selon le rapport qu'ils entretiennent vis-à-vis de la commande : à l'ouest se retrouvent des artistes qui développent essentiellement un travail d'atelier dans une posture d'artiste autonome dont l'œuvre préexiste à son marché ; à l'est, on retrouve les artistes qui, au contraire, inscrivent une partie substantielle de leur activité dans une réponse à une demande privée ou institutionnelle. Les variables qui influencent le plus cet axe sont la formation en école d'art appliqué, être sculpteur, exercer un autre métier non artistique, gérer un lieu ou une association.

Au final, quatre groupes d'artistes ont été identifiés, les deux premiers tournés vers la tradition (l'artiste de salon, l'artiste artisan-entrepreneur), les autres vers l'innovation (l'Art fair artist' ou artiste de foire et l'artiste à $360^{\circ}$ ). La première ligne de partage nord/ sud confirme donc l'hypothèse de profils distincts d'artistes selon le type d'art pratiqué (Caves, 2000 ; Galenson, 2000 ; Moureau, Sagot-Duvauroux, 2010), profils qui mettent en évidence le caractère discriminant de la formation.
Nous détaillons dans les parties suivantes les caractéristiques des différents profils identifiés.

\section{L'ancrage dans la tradition: de l'artiste de salon à l'artiste artisan entrepreneur}

Comme il a été mentionné, une ligne de partage innovation/tradition se dessine entre les parties nord et sud du graphique. Dans le cadran sud, différentes variables permettent de caractériser les artistes de tradition. «Autodidactes » ou ayant eu une formation en école « d'art appliqué », ils produisent un art dans le sens classique du terme, i.e. de la peinture pour les 《 artistes de salon » et plutôt de « la sculpture » pour les « artistes artisans entrepreneurs ».

\section{L'artiste de salon}

« L'artiste de salon » produit un art de tradition. Nous avons là l'image classique du « peintre ». Sa production, constituée pour l'essentiel de tableaux, est diffusée en « galerie privée », un même artiste pouvant faire appel à plus d'une dizaine de galeries. Les plus connus disposent d'un réseau « international».

Peu d'acteurs interviennent dans la chaîne qualifiante ; la qualité du travail est avant tout endogène à l'œuvre et par conséquent directement appréciable par le collectionneur lui-même. L'intermédiation institutionnelle est quasi-inexistante dans la carrière de l'artiste, elle est peu nécessaire puisque la qualité de l'œuvre est en principe directement lisible. Ses œuvres sont exposées dans des « salons » ainsi que dans des « foires ». Les prix décrochés lors de « salons » constituent des signaux importants de reconnaissance pour les collectionneurs. Ceux-ci s'intéressent souvent à un genre (les marines, les nus), à une école et peuvent être d'une grande érudition dans la spécialité de leur collection.

Les données qualitatives recueillies lors des entretiens semi-directifs auprès des acteurs du marché de l'art précisent ce premier portrait. Les galeries s'appuient sur les recommandations émanant des autres artistes de la galerie dans leurs sélections, soulignant ainsi l'importance de s'inscrire dans des réseaux d'artistes, quel que soit le type d'art pratiqué ; quant à certaines revues spécialisées et sites Internet, ils apparaissent 


\section{Graphique 1}

\section{Répartition des artistes en quatre groupes}

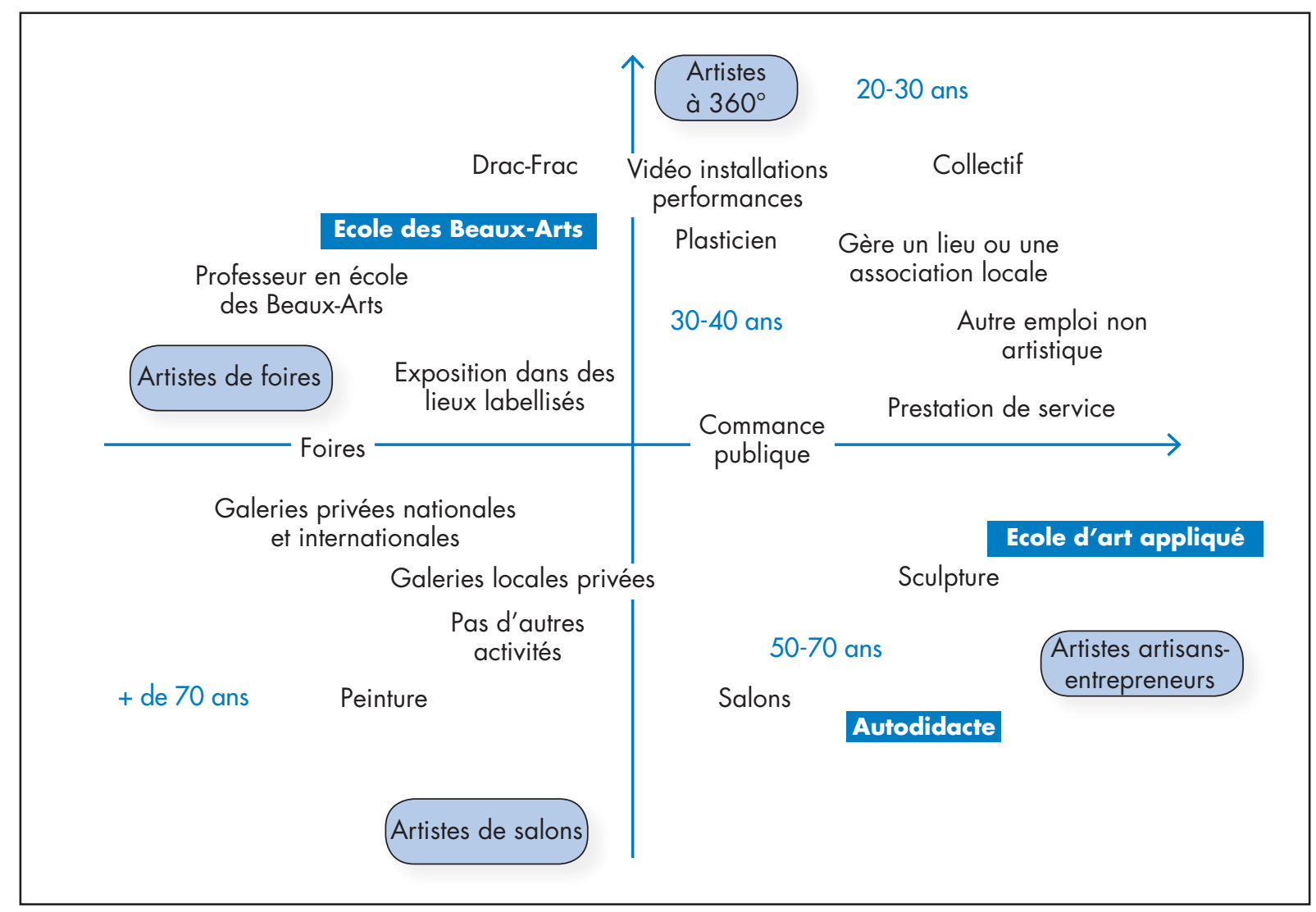

Source : Etude réalisée pour le DEPS, ministère de la Culture, disponible sur le site du ministère : Culture études 2011.1

\section{Note de lecture :}

L'analyse factorielle des correspondances ci-dessus est la projection dans un plan d'un tableau croisé où figurent en ligne les artistes interrogés et en colonne les variables qui les caractérisent. Ne sont mentionnées sur le graphique que les variables importantes dans notre typologie. Se retrouvent à proximité dans le plan les artistes qui ont des caractéristiques voisines au regard des variables qui les définissent. L'analyse factorielle fait ainsi apparaître des groupes d'artistes sur lesquels se fonde notre typologie et qui apparaissent en grisé. L'axe vertical oppose les artistes « classiques » (au sud) aux artistes qui s'inscrivent dans la convention d'originalité (au nord). Les variables DRAC-FRAC, plasticien, école des beaux arts (au nord) s'opposent aux variables peinture, salons, autodidacte au sud. L'axe horizontal distingue, à gauche, les artistes qui pratiquent une économie d'œuvres à ceux, à droite, qui pratiquent davantage une économie de projet. Les variables foires, peinture, galeries privées à gauche s'opposent aux variables sculpture, prestation de service gestion d'un lieu ou d'une association à droite.

\section{Variables :}

- Formation : Ecole des beaux arts, Ecole d'art appliquée, Autres écoles d'art, Autodidacte.

- Age : 20-30 ans, 30-40 ans, + de 70 ans.

- Medium pratiqué : Peinture, Sculpture, Photo, Vidéo installations performance, Plasticien.

- Lieux d'expositions : galeries locales privées, galerie locale associative, galeries privées nationales et internationales.

- Activités secondaires : professeur dans une école des beaux-arts, professeur dans une école d'art appliqué, autre emploi non artistique, pas d'autres activités.

- Soutien institutionnel : Drac, Frac, Exposition dans des lieux labellisés.

- Participation collectives : salons, foires.

- Implication sur le territoire : Gérer un lieu ou une association locale, appartenir à un collectif, répondre à une commande publique. 
comme des outils importants de découverte et de promotion des « artistes de salons », non pas tant en raison des discours qui s'y développent qu'en raison de l'existence de reproductions qui permettent au galeriste ou au collectionneur de visualiser la qualité du travail. Les sources de revenus des ces artistes proviennent essentiellement des ventes d'œuvres, mais aussi parfois de produits dérivés (cartes, affiches pour des évènements). "L'artiste de salon » peut avoir des activités complémentaires comme des cours privés. Sa production est majoritairement autonome ; elle répond peu à des commandes ou à des appels à projets.

Ainsi, M2, né en 1936, a commencé par être céramiste et reçoit la médaille d'argent du meilleur ouvrier de France. Comme son travail de céramiste «l'ennuie », il se forme sur le tas comme staffeur décorateur. Il peint en parallèle et suit « un peu les cours du soir de la ville de Paris ». En 1960, il participe à ses premiers salons. Il obtient alors de très nombreux prix (62, premier prix ville d'Avray, 63 Clamart, 64 Chatenay, 66, APPAS). En 68, il reçoit la médaille d'argent des arts sciences et lettres et en 73, il est nommé sociétaire du salon d'automne. Les médailles se succèdent (Médaille d'or du salon des artistes Français en 1978. Salon des Peintre Témoins de Leur temps en 1982, grand Prix de Barbizon) jusqu'à ce qu'en 1983, il soit nommé peintre officiel de la marine. Ses premières participations à des salons lui ont rapidement permis d'être repéré par des galeries qui l'exposent. Jusqu'en 1980, ses expositions personnelles se déroulent en France; puis, à partir de cette date, il expose très régulièrement à l'étranger dans des galeries américaines mais il a eu également quelques expositions au Japon et à Londres. L'artiste déclare avoir gagné beaucoup d'argent à une époque. Il connait actuellement une crise de production «depuis deux ans, je n'ai quasiment rien sorti. J'ai envie d'autre chose ». "Je n'ai pas envie de me répéter mais cela pose problème avec mon marchand des États Unis, cela fait treize ans que je travaille avec lui » «j'ai envoyé mon nouveau travail. Mais personne n'en a voulu ». En France, il est exposé par deux galeries privées, il n'a jamais eu de soutien des institutions.

\section{L'artiste artisan entrepreneur}

« L'artiste artisan-entrepreneur » constitue un deuxième modèle d'artiste de tradition. Les artistes se déclarant « sculpteurs » correspondent bien à ce profil. Compte tenu du coût de leur medium, ils doivent trouver des sources de financement et leur travail s'inscrit assez facilement dans le paysage urbain ; cela leur permet d'être mieux placés que d'autres sur « des commandes publiques » de communes ou d'agglomérations. Plutôt âgés [50,70], ils peuvent avoir été formés auprès d'un « maître », dans « une école d'art appliqué » ou bien être « autodidactes ». Peu d'intermédiaires interviennent dans leur parcours car ils démarchent souvent eux-mêmes les différentes institutions. La seule intermédiation passe par des sites qui recensent les appels à projets et les services des collectivités publiques (aides au dossier, requalification de l'œuvre si besoin), ainsi que les sites d'artistes qui proposent une aide. Mais l'appartenance à des réseaux proches des pouvoirs politiques locaux peut être un élément déterminant du succès

L'examen des entretiens révèle que ces artistes adoptent souvent une démarche d'entrepreneurs. Ils s'entourent parfois d'une équipe, d'un agent et éventuellement de secrétaires qui assurent les tâches administratives liées au développement de l'activité. Ils peuvent déléguer une partie de la réalisation si celleci exige des compétences qu'il ne possède pas ou rémunérer un salarié si la demande est élevée ou s'il travaille sur un projet monumental. La valorisation du travail passe par des réponses à des appels d'offre ou par le démarchage d'entreprises privées. Les lieux de diffusion sont souvent des espaces ouverts au public.

Les revenus de ces artistes proviennent de facturations de commandes, de travaux sur devis. L'artiste de commande peut également exercer des activités complémentaires ; cependant, celles-ci restent marginales, comme la perception de droits d'auteurs, ou quelques cours privés. Finalement, ces artistes perpétuent la tradition de l'artiste-artisan des corporations d'antan.

Ainsi, N1 est un parfait self made man, bien qu'ayant eu une enfance baignée dans les œuvres d'art avec un père collectionneur. Faisant profession de maître d'armes, il pratique la sculpture 
dans ses moments de loisirs jusqu'à ce qu'il rencontre un sculpteur-restaurateur, avec qui il va collaborer comme artisan pendant trois ans. Son expérience de conseiller municipal lui permet de mieux connaître le fonctionnement des collectivités locales, expérience précieuse lorsqu'il décide de se mettre à son compte. Il fait alors le tour des mairies pour obtenir des commandes et expose ses œuvres dans des salles de fêtes. Adhérent à la Maison des Artistes depuis 11 ans, il reconnaît que ses indemnités d'élu représentent plus de la moitié de ses revenus. Il passe $50 \%$ de son temps sur internet pour consulter les appels à candidature. Il participe aussi à des symposiums de sculptures. Ils consistent à inviter une dizaine de sculpteurs pendant une période de temps donné durant laquelle ils sont payés à réaliser une œuvre in situ, les œuvres étant, dans certains cas, conservées par la ville, d'autres fois restituées à l'artiste.

\section{Les artistes tournés vers l'innovation: de l'Art Fair Artist à l'artiste à $360^{\circ}$.}

Les artistes présents dans les cadrans nord du graphique 1 ont pour caractéristique commune d'être tournés vers un art de recherche. Ils ont généralement reçu une formation dans une «école des beauxarts ». Ce sont des « plasticiens » qui n'hésitent pas à travailler avec les nouvelles technologies, pratiquant pour certains un « art vidéo ». Nous présenterons deux variantes de l'artiste chercheur : l'artiste de foires et $l^{\prime}$ 'artiste à $360^{\circ}$. Dans cette dernière catégorie, nous distinguerons le profil particulier de l'artiste acteur de la vie artistique locale.

\section{L'Art Fair Artist}

L'Art fair artist est le pendant de l'artiste de salon versus recherche. Comme ce dernier, il s'inscrit dans une " économie d'œuvres », d'objets appropriables susceptibles d'entrer dans une collection. Généralement formé dans une école des " beauxarts », il diffuse son travail en « galeries privées nationales ou internationales » mais il est également proche du réseau institutionnel (FRAC, DRAC) et ses œuvres sont exposées dans des « lieux labellisés ». L'intermédiation est mixte, à la fois marchande et institutionnelle. Les commissaires d'expositions, les centres d'art, les FRAC contribuent, par leur reconnaissance, à réduire l'incertitude qualitative qui entoure le travail d'un jeune artiste. Leurs choix sont analysés comme autant de signaux de qualité susceptibles d'engendrer une cascade informationnelle ${ }^{5}$, relayée ensuite par les galeries et les collectionneurs. L'intermédiation est donc qualifiante.

Ici encore, la lecture des entretiens permet de préciser ce portrait robot. D'une façon générale, à la sortie de l'école, grâce aux réseaux constitués pendant ses études, l'Art fair artist obtient une exposition dans une galerie associative, souvent gérée par un ancien de l'école. Parfois, il prend lui-même en main son destin en créant un collectif d'artistes qui attire davantage l'attention qu'un artiste isolé. Par la suite, le soutien apporté par une exposition dans un centre d'art labellisé et, mieux encore, l'achat par un FRAC, peuvent lui ouvrir les portes d'une galerie parisienne en prise avec le marché national et international de l'art. La présentation ou l'achat de son travail dans un musée reconnu et une présence dans une grande foire consacreront sa carrière. La valorisation du travail de l'Art Fair Artist s'effectue par l'intermédiaire de galeries de promotion. La réussite de l'artiste se mesure à l'aune de la présence de ses œuvres dans les grands musées, les grandes collections privées, les foires et biennales internationales, et à travers la publication d'un discours critique sur son travail dans une revue spécialisée (Art Press, etc.).

Ses principales sources de revenus proviennent de la vente d'œuvres, mais aussi de l'enseignement dispensé aux beaux-arts. Car une des meilleures façons de faire fructifier une notoriété naissante, est d'obtenir un poste dans une école d'art qui protègera plus tard l'artiste contre le risque, très réel, d'une chute de notoriété. Les artistes qui visent un parcours ayant pour objectif une intégration au marché international de l'art contemporain doivent s'attendre à une carrière chaotique. Chaque nouvelle génération d'ar-

\footnotetext{
Une cascade informationnelle survient lorsqu'il est optimal pour un individu ayant observé les actions des autres avant lui, de suivre le comportement des agents qui le précèdent, sans tenir compte de sa propre information. Cette dynamique mimétique peut conduire rapidement à des effets pervers si les premiers entrants sur le marché font un choix erroné ou stratégique (c'est-à-dire un choix qui anticipe les effets mimétiques de ce choix à des fins opportunistes. Les bulles spéculatives peuvent être le résultat de cascades informationnelles. Cf. Bikhchandani et alii (1992; 1998)).
} 
tiste a tendance à déclasser les artistes plus anciens qui n'ont pas réussi à accéder à une position incontournable sur le marché.

Ainsi, R1 a 37 ans ; il est diplômé d'école d'art. Deux ans après sa sortie de l'école, il bénéficie d'une aide à la création de la DRAC ; à cette occasion, il rencontre le directeur du FRAC qui lui propose une exposition collective « jeune création ». À l'issue de cette exposition, ses contacts se multiplient : musée des beaux-arts de Rouen, Galerie rouennaise. Après une exposition personnelle dans un centre d'art allemand, plusieurs expositions dans les centres d'art régionaux et deux achats FRAC, il rencontre un galeriste parisien qui lui propose une exposition collective. Il vend bien, continue d'exposer dans des lieux institutionnels (centres d'art, musées des beaux-arts du Mans, Musée en Belgique) et devient un artiste « permanent» de la galerie qui l'expose dans les foires. Il est directeur d'école d'art municipale.

\section{L'artiste à $360^{\circ}$}

Plus jeune en moyenne (la moitié a moins de 40 ans) que l'Art Fair Artist (un quart a moins de 40 ans), l'artiste à $360^{\circ} \mathrm{s}$ 'en distingue également par une volonté plus affichée de répondre à la demande sociale en valorisant ses compétences créatives sous des formes qui ne prennent pas nécessairement celle d'une œuvre appropriable et collectionnable. Il répond, comme l'artiste-entrepreneur-artisan, à des commandes, à des appels à projets, mais s'en écarte par des créations du type performances, installations, « vidéos ». En ce sens, il s'inscrit davantage dans la recherche plastique contemporaine. Sa formation a lieu dans des écoles des beaux-arts. Il appartient souvent à un « collectif» d'artistes ; parfois, il se définit comme prestataire de services de création pour les collectivités ou les entreprises (réponses à des appels d'offres au côté d'architectes, commissariat d'expositions, formation...). L'intermédiation institutionnelle est forte, l'intermédiation marchande plus modérée car cet artiste ne passe pas forcément par des galeries pour exposer ses œuvres.

Le rôle fort des institutions pour leur carrière tient à ce qu'elles sont des pourvoyeuses d'informations et constituent la principale source de financement de ces artistes. On peut ici véritablement parler d'économie institutionnelle. D'où une forte vulnérabilité de ces artistes au changement du conseiller art plastique de la DRAC ou du directeur du FRAC ; ce changement peut en effet se traduire par une marginalisation rapide de l'artiste si son travail ne rencontre plus l'intérêt du nouveau responsable. Les modes de valorisation de l'œuvre de l'artiste à 360 sont multiples : il vend des œuvres (un peu), répond à des appels d'offre, intervient dans les écoles comme médiateur de l'art contemporain, dépose des dossiers de résidence, joue un rôle de commissaire d'exposition. Les lieux de diffusion du travail sont par conséquent pluriels : les galeries, les espaces ouverts au public, les entreprises. La multiplicité des partenaires implique que la réussite se mesure en partie par la grandeur et la diversité du réseau au sein duquel il s'insère. Le degré de centralité du réseau, mesuré par ne nombre de relations qu'entretient l'artiste avec les autres membres du réseau, constitue un indicateur de sa réussite. Mais comme la valeur de l'artiste tient à sa place dans un réseau très ancré territorialement, il rencontre souvent des difficultés à s'exporter hors de sa région.

Ainsi, A6, né en 1974, est diplômé de plusieurs écoles : diplôme national d'art plastiques à Nancy, diplôme national supérieur d'expression plastique à Paris et post diplôme à Lyon en 2001. L'année de son post diplôme, il expose au MAC (musée d'art contemporain) de Lyon « Rendez vous » et au CNP (Centre national de la photographie) à Paris. Il expose depuis principalement dans des lieux alternatifs à Lyon. En 2007, il expose dans une galerie associative lyonnaise pendant la biennale, ce qui lui « vaut de nombreux contacts » et lui permet de bénéficier d'une résidence d'artiste. Il obtient une aide à la création de la Drac Ile de France en 2003 ainsi que celle du DICRéAM (dispositif pour la création artistique multimédia, aide accordée par le Centre national de la cinématographie Délégation aux Arts Plastiques) pour deux films. Il n'est pas représenté par une galerie. À propos de ses revenus, il dit: " Je n'ai pas fait de ventes, ni de locations, ni de prix dans le cadre de festivals, j'ai une activité de salarié en quelque sorte : je fais beaucoup de workshops (d'ateliers) et de travaux pour des entreprises ... » Ces activités constituent son principal revenu. Il habite à Paris car « il y a 
peu de résidences en région Rhône-Alpes et Paris permet plus de rencontres » mais travaille régulièrement avec les lieux d'art contemporain de la Région Rhône-Alpes.

\section{$L^{\prime}$ artiste à $360^{\circ}$ animateur de la vie artistique locale}

Il constitue une variante de ce profil. Le créateur, pour des raisons stratégiques (renforcer sa place dans les réseaux) et/ou politique et militante (inscrire la création artistique dans la réalité sociale de la ville) ne se contente pas de développer son activité artistique dans de multiples directions ; par la gestion d'une galerie, la création d'une association d'action artistique, la réalisation de projets inscrits dans le territoire, il met ses compétences au service d'autres artistes, d'acteurs territoriaux ou des populations locales. S'établissent ainsi des réseaux d'artistes souvent auto-organisés sous forme de collectifs, qui prennent leur destin en main face à la difficulté qu'ils rencontrent à intégrer les réseaux existants. Ils construisent ainsi leur propre réseau, très ancré territorialement.

Ainsi, N7 et N8 sont tous deux titulaires d'un diplôme d'une école des beaux-arts. Ce couple d'artistes développe, depuis une vingtaine d'années, une activité de création, le plus souvent au sein de collectifs d'artistes qui ne séparent pas leur activité d'artiste de celles de médiateur, programmateur, intermédiaire de l'art contemporain. Ils s'appuient sur les dispositifs existants pour diversifier les modes d'intervention des artistes. Ils sont commissaires d'exposition ou simplement régisseurs ; ils organisent le brassage international de l'art à travers l'organisation de voyages de travail, procèdent à des échanges d'expositions avec des artistes ou des lieux croisés lors de ces voyages; ils gèrent un site internet au service d'une quarantaine d'artistes régionaux sélectionnés par un comité d'experts. En collaboration avec un cabinet d'architecte, ils répondent à des appels d'offre. Enfin, ils assument un rôle de médiateur auprès des écoles pour diffuser l'art contemporain.

\section{LE RÔLE DIFFÉRENCIÉ DE LA FORMATION SELON LE TYPE DE CRÉATION}

L'analyse factorielle et les profils type d'artistes qui s'en dégagent mettent en évidence un lien fort entre type de création et formation. Dans le monde de l'art contemporain, le passage par une école des beaux-arts est quasiment incontournable, tandis que les artistes engagés dans un art de tradition ont recours soit à une auto-formation, soit à des écoles privées, soit encore à un apprentissage auprès d'un maître. Ainsi, sur les 44 artistes de notre échantillon qui sont passés par l'école des beaux-arts, 38 appartiennent au monde de l'art contemporain. Les six restants sont des artistes relativement âgés dont le passage par l'école a précédé la réforme de celle-ci. À l'inverse, sur les 17 artistes autodidactes, 13 appartiennent aux catégories artistes de salons et artistes entrepreneurs.

Le rôle différencié de la formation, selon le monde de l'art auquel on se réfère, renvoie à l'économie de chacun de ces mondes et, en amont, aux conventions de qualité auxquelles ces mondes se réfèrent.

\section{Le monde de l'art contemporain}

Ici, la construction de la valeur marchande est largement endogène à la façon dont l'œuvre entre sur le marché. La valeur ne s'ancre pas tant sur les caractéristiques intrinsèques de l'oeuvre (technique utilisée, taille, capacité émotive, agencement des couleurs, etc.), même si ces critères ne sont pas totalement absents, que sur la façon dont elle est située, par les acteurs de ce monde, dans une histoire de l'art en train de se faire. Comme le note Nathalie Heinich, « cet art contemporain qui a tant élargi les frontières de l'art n'est accessible qu'à ceux qui ont réussi à rentrer dans ce monde aux frontières bien délimitées, dans lequel on ne pénètre plus par la contemplation des objets (...) mais par les récits qui les trament, c'est-àdire par les personnes qui les racontent $\gg{ }^{6}$

Ce processus commence dès l'Ecole des beaux-arts. Bénédicte Martin (2005) a montré comment l'étudiant des beaux-arts apprend à construire un discours sur

6 Heinich, N., Idem, p. 197. 
Tableau 2

Type d'artiste selon le médium utilisé, la formation et le soutien institutionnel

\begin{tabular}{|l|c|c|c|c|c|c|c|c|}
\cline { 2 - 9 } & Nombre & Peintres & Sculpteurs & $\begin{array}{c}\text { Plasticiens, } \\
\text { photographes, } \\
\text { vidéastes, } \\
\text { performers }\end{array}$ & $\begin{array}{l}\text { Formation } \\
\text { dans une } \\
\text { école des } \\
\text { beaux-arts }\end{array}$ & Autodidacte & Autre & $\begin{array}{c}\text { Soutien } \\
\text { institutionnel }\end{array}$ \\
\hline Art Fair artists & 27 & 11 & 2 & 14 & 22 & 2 & 3 & 24 \\
\hline Artistes $360^{\circ}$ & 20 & 0 & 0 & 20 & 16 & 3 & 1 & 19 \\
\hline $\begin{array}{l}\text { Artistes } \\
\text { de salon }\end{array}$ & 15 & 13 & 2 & 0 & 5 & 8 & 2 & 0 \\
\hline $\begin{array}{l}\text { Artistes artisan } \\
\text { entrepreneur }\end{array}$ & 7 & 2 & 5 & 0 & 1 & 4 & 2 & 2 \\
\hline Total & 69 & 26 & 9 & 34 & 44 & 17 & 8 & 45 \\
\hline
\end{tabular}

Note de lecture : Sur 69 artistes au total, 27 correspondent au profil 'Art Fair artist'. Parmi ces derniers, 11 pratiquent de façon privilégiée la peinture, 2 la sculpture et 14 travaillent plusieurs média ou se déclarent comme plasticiens. S'agissant de leur formation, 22 ont été formés dans une école des beaux arts, 2 sont autodidactes et 3 ont reçu un autre type de formation. Enfin, sur l'ensemble de ces 27 artistes, 24 ont bénéficié d'un soutien institutionnel (e.g. bourse de création).

Source : Etude réalisée pour le Département des Etudes, de la Prospective et de la Statistique DEPS ministère de la Culture. Synthèse de l'étude disponible sur le site du ministère : Culture études 2011.1

son œuvre et est évalué sur la cohérence qu'il sait lui conférer. Il le fait avec le soutien de ses professeurs, qui sont les premiers intermédiaires de son travail. Outre une aide à la construction de ce discours sur l'œuvre, les professeurs (souvent critiques ou artistes) ouvrent la porte aux premiers relais institutionnels indispensables à la reconnaissance artistique du travail, qu'il s'agisse d'un commissaire d'exposition dans un centre d'art, d'un membre d'une commission d'achat (Fonds régional d'art contemporain), d'un animateur d'une galerie associative ou encore d'un artiste représenté par une galerie. Le réseau social du professeur, autant que la capacité du jeune artiste à s'approprier le réseau de son maître et à construire le sien propre constituent des éléments déterminants de la réussite de cette première épreuve.

En ce sens, la réforme des écoles des beaux-arts, au début des années 80 , a bien répondu aux évolutions du marché. Elles ont en effet modifié leur pédagogie afin de corriger les excès de la libéralisation totale de 1968. Celle-ci avait instauré une trop grande autonomie accordée aux élèves qui s'avérait préjudiciable pour leur insertion sur la scène internationale. Afin de favoriser la professionnalisation des élèves, les écoles ont cherché à s'ajuster aux principes en vigueur sur le marché international de l'art contemporain. Deux axes ont servi cette nouvelle politique : l'entrée des nouvelles technologies à l'école et une ouverture de l'école vers le monde de l'art et ses réseaux. Dans les recherches qu'elle avait conduites à la fin des années 90, Françoise Liot avait montré l'impact de l'école des beaux-arts sur les pratiques artistiques, lié au développement d'un art discursif et aux modes de sélection et d'attribution des bourses (Liot, 1999).

Les entretiens réalisés, tant auprès des artistes que des acteurs de l'art, confirment ce processus. Les écoles des beaux-arts jouent un rôle essentiel dans la structuration des mondes de l'art contemporain au sein des agglomérations enquêtées ; ce rôle s'exerce à travers les réseaux de leurs professeurs, à travers les liens construits avec les « anciens » de l'école qui se retrouvent à la tête d'un centre d'art local ou sont intégrés à des galeries dont ils peuvent faciliter l'accès.

Autrement dit, les réseaux auxquels donne accès une école des beaux-arts n'ont pas pour seul rôle de faciliter la visibilité des étudiants de ces écoles de façon à ce que le marché puisse ensuite opérer sa sélection 
sur la base d'une qualité supposée exogène. Ils contribuent à construire la qualité même de l'œuvre, qui est indissociable du discours qui la justifie, la situe et la classe.

\section{Le monde de l'art classique}

Il en est tout autrement du monde de l'art classique. Dans le prolongement des conventions esthétiques de l'académie, dont les critères d'évaluation de la qualité définis par Roger de Piles (1766) fournissent une bonne idée, la qualité des œuvres est intrinsèque à l'œuvre ; soit que cette qualité se réfère à un registre émotionnel, soit qu'elle renvoie à des critères purement techniques (qualité de la facture par exemple). Tandis que la connaissance du nom de l'auteur est absolument indispensable pour évaluer la qualité d'une œuvre dans le monde contemporain, il n'est pas indispensable dans le monde de l'art classique.

Ici, le collectionneur n'attend pas du critique ou de la galerie qu'ils construisent un discours sur l'œuvre mais plus simplement qu'ils opèrent un travail de sélection des artistes en cohérence avec les critères de qualité en vigueur. Le rôle de la formation est alors de fournir à l'artiste un savoir-faire lui permettant d'exprimer au mieux son talent, à charge ensuite au marché d'opérer la sélection.

Bien sûr, les réseaux jouent un rôle, mais qui est de nature différente. L'existence de communautés d'artistes autour d'un genre (le nu, les marines) favorise la visibilité. Dans un marché qui fonctionne largement par recommandation, ces réseaux multiplient les possibilités d'être signalé ou recommandé. Mais ils ne construisent pas en tant que telle la valeur.

Si certains artistes de salons, les plus âgés, ont pu suivre une formation en école d'art, c'est alors parce qu'à l'époque de leur formation, les écoles d'art ne s'étaient pas encore adaptées aux réalités du marché de l'art international. L'examen des entretiens conduits auprès de ces artistes montre que ce sont souvent des artistes qui ont eu dans le passé leur heure de gloire en étant des figures d'un mouvement artistique qui a compté. Ils ont été formés dans une école des beaux-arts au sein de laquelle ils sont parfois devenus professeurs ; cependant, leur mouvement est passé de mode et n'intéresse plus les institutions qui les avaient reconnus à un moment donné de leur carrière. Les Fonds régionaux d'art contemporain sont trop récents pour s'être intéressé à leur travail ; toutefois, il leur est possible d'exposer des œuvres dans les musées municipaux. Ils ont glissé du monde de

Tableau 3

Profil des artistes sortis de l'école des beaux-arts en fonction de leur âge

\begin{tabular}{|l|c|c|c|c|c|c|c|c|}
\cline { 2 - 9 } & $20-30$ & $30-40$ & $40-50$ & $50-60$ & $60-70$ & $70-80$ & $80-90$ & Total \\
\hline Art Fair & 2 & 4 & 6 & 8 & 1 & 1 & & 22 \\
\hline Artistes à 360 & 1 & $6^{*}$ & 9 & & & & & 16 \\
\hline Salon & & 1 & & & 1 & & 3 & 5 \\
\hline Entrepreneurs & & & & & 1 & & & 1 \\
\hline Total & 3 & $11^{* *}$ & 15 & 8 & 3 & 1 & 3 & 44 \\
\hline
\end{tabular}

Source : Etude réalisée pour le DEPS, ministère de la Culture. Synthèse de l'étude disponible sur le site du ministère : Culture études 2011.1

Note de Lecture : Sur le total des 44 artistes de l'échantillon ayant reçu une formation à l'école des beaux-arts, $11^{* *}$ ont entre 30 et 40 ans. Sur ces onze artistes, $6^{*}$ correspondent au profil «Artistes à $360^{\circ} »$. 
l'art contemporain vers celui de l'art moderne. «Par rapport aux musées, aux galeries et critiques, j'ai eu d'abord de la chance et après cela s'est gâté. Quand je faisais mes expositions au musée d'art moderne de la ville de Paris, mes toiles étaient accrochées dans les mêmes salles que Desnoyer. Puis la dégringolade $s$ 'est produite. Une salle, une cimaise pour Desnoyer, puis plus rien du tout. Cela a été la même chose pour $m o i »$ déclare un artiste.

Précisons que dans les agglomérations enquêtées (Le Havre, Rouen, Lyon Montpellier, Nantes), les écoles d'art ne jouent aucun rôle dans la structuration des réseaux de l'art classique.

Cet article met en évidence le lien qui existe entre le mode d'apprentissage du métier d'artiste, le monde de l'art dans lequel il s'inscrit et les conventions esthétiques qui régissent ce monde.

Dans le monde de l'art classique (« artiste entrepreneur » et « artiste de salon »), la reconnaissance passe prioritairement par le marché. La qualité est intrinsèque à l'œuvre et le collectionneur est en mesure, par lui-même, en fonction de la qualité technique de la réalisation et du sujet, de porter son propre jugement. La formation a pour rôle l'apprentissage des techniques. Elle se fait chez un maître ou dans une école spécialisée. L'intermédiation est faible.

A contrario, dans le monde de l'art contemporain tourné vers l'innovation ( « art fair artist », « artiste à $\left.360^{\circ} »\right)$, l'artiste tire sa notoriété de la reconnaissance par des instances de légitimation de l'originalité de sa démarche. Il doit apprendre à construire un discours sur son œuvre et à convaincre quelques intermédiaires (galeries commissaires d'expositions, FRAC) de la pertinence de son travail pour qu'à leur tour, ils développent un discours critique apte à faire accepter l'artiste dans les réseaux internationaux.

Grâce aux réformes engagées dans les années 80 , l'École des beaux-arts a pu endosser ce double rôle : apprendre à construire ce discours visant à souligner le caractère innovant de l'oeuvre (« convention d'ori- ginalité ») ; faciliter l'accès aux « instances de légitimation de l'art » par l'intermédiaire notamment du réseau de relation du corps professoral. Une carrière réussie suit alors les étapes suivantes : à la sortie de l'Ecole des beaux-arts, l'artiste participe à une exposition collective organisée par une galerie associative locale, souvent tenue par un ancien de l'École. Cette exposition attire l'attention des centres d'art contemporain de la région ainsi que du FRAC. La reconnaissance conférée par ces instances (achat, exposition) éveille à son tour l'intérêt d'intermédiaires en lien avec ces instances locales et ayant un rayonnement national (galeries parisiennes, commissaires d'expositions, musées, etc.). À leur tour, ces acteurs attirent l'attention d'intermédiaires internationaux, etc. Le mythe de l'artiste libre et indépendant est rattrapé par la réalité.

Rappelons enfin que la typologie d'artistes dégagée est issue d'une enquête ne visant pas la représentativité statistique. Il s'agissait d'identifier, à partir des entretiens, des profils emblématiques émanant des différents mondes de l'art. Cette enquête nous a permis de repérer les variables les plus discriminantes. Un prolongement de ce travail serait alors de construire une enquête auprès d'un échantillon statistiquement représentatif d'artistes. Il s'agirait de mesurer le poids des différents types identifiés dans la population totale et de préciser leurs caractéristiques.

Au-delà, cet article montre que la formation est un élément déterminant des mondes de l'art et conditionne fortement la carrière future des artistes. Reste à savoir si les choix esthétiques des jeunes artistes précèdent le choix de leur formation, ou si c'est le type de formation reçue qui conditionne les choix esthétiques. Autrement dit, il importe de savoir dans quelle mesure les artistes demeurent libres du choix du type d'art qu'ils pratiquent. 
Alper N. O., Wassall G. H. (2006), “Artists' Careers and their Labor Markets", in Ginsburgh V. A., Throsby D., Handbook of the Economics of Art and Culture, Elsevier North Holland, pp. 814-864;

Arrow K. (1973), "Higher Education as Filter", Journal of Public Economics, 2, pp. 193-216.

Becker G. (1964), Human capital. A theoretical and Empirical Analysis with Special Reference to Education, The University of Chicago Press, Chicago.

Becker H. (1988), Les mondes de l'art, Flammarion (ed originale 1982).

Benhamou F. (2004), L'économie de la culture, Repères, La Découverte.

Bikhchandani S., Hirshleifer D., Welch I., (1992) “A theory of fads, fashion, custom and cultural change as informational cascades", Journal of Political Economy, ${ }^{\circ} 5$, vol. 100, october.

Bikhchandani S., Hirshleifer D., Welch, I. (1998), "Learning from the behaviour of others ; conformity fads and informational cascades", Journal of Economic Perspectives, vol. 12, n 3, pp. 151-170.

Bielby D. (2009), "Gender Inequality in Culture Industry:Women and Men Writers in Film and Television", Sociologie du travail, 51 (2), pp. 237-252.

Blaug M. (1985), "Where Are We in Economics of Education?", Economics of Education Review, 4 (1), pp. 17-28.

Buscatto M. (2007), "Women in Artistic Professions. An Emblematic Paradigm for Gender Studies", Social Cohesion and Development Journal, 2(1), pp. 69-77.

Caves R. (2000), Creative Industries: Contracts Between Art and Commerce, Cambridge Mass./ London, Harvard University Press.
De Piles R. (1766), Cours de Peinture par Principes, Kessinger Publishing, september.

De Vriese M., Martin B., Melin C., Moureau N., Sagot-Duvauroux D. (2011), Diffusion et valorisation de l'art actuel en région, Cultures Etudes, CE-201101, DEPS, ministère de la Culture.

Galenson D. (2000), “Age and the Quality of Work: the Case of Modern American Painters", Journal of Political Economy, 108, pp. 761-777.

Galenson D (2000), "Careers of Modern Artists: Evidence from Auction of Contemporary Art", Journal of Cultural Economics, 24, pp 87-112.

Heinich N. (1993), Du peintre à l'artiste, artisans et académiciens à l'âge classique, Paris, Les éditions de Minuit.

Heinich N. (1998), Le triple jeu de l'art contemporain, Paris, Les Éditions de Minuit.

Heinich N. (1999), Pour en finir avec la querelle de l'art contemporain, Paris, LEchoppe.

Liot F. (1999), « L'école des Beaux-arts face aux politiques de soutien à la création », Sociologie $d u$ travail, 41, pp 411-429.

Martin B. (2005), L'évaluation de la qualité de l'art contemporain Le cas de l'insertion des jeunes artistes plasticiens. Thèse de doctorat en sciences économiques, Université Paris X.

Menger P.-M., (2006), “Artistic Labor Markets : Contingent Works, Excess Supply and Occupational Risk Management", in Ginsburgh V. A., Throsby D., Handbook of the Economics of Art and Culture, Elsevier North Holland, pp 766-811.

Menger P.-M. (2009), Le travail créateur, Gallimard, Seuil. 
Menger P.-M. (2010), "Les artistes en quantité, ce que sociologues et économistes s'apprennent sur le travail et les professions artistiques", Revue d'Economie Politique, 120 (1), pp 205-236.

Mingat A., Eicher J.-C. (1975), Education, inequality and life chances, Paris, OCDE.

Moulin R. (1992), L'artiste, l'institution et le marché, Flammarion.

Moureau N., Sagot-Duvauroux D. (2010), Le marché de l'art contemporain, La Découverte.

Perrenoud M. (2008), « Les musicos au miroir des artisans du bâtiment : entre « art » et « métier»", Ethnologie française, 38 (1), pp. 101-106.

Rosen S. (1987), "Human Capital", in Eatwell J., Milgrate M., Norman P. (eds.), The New Palgrave: A Dictionary of Economics, Macmillan, London and Basingstoke, pp. 681-689.
Rosen S. (1981), "The Economics of Superstars", American Economic Review, 71 (5), 845-858.

Spence M. (1973), "Job Market Signaling”, The Quarterly Journal of Economics, 87(3), pp. 355-374.

Thornton S. (2008), Sept jours dans le monde de l'art, Autrement.

Towse R. (2006), “Human Capital and Artists'Labour Markets", in Ginsburgh V. A., Throsby D., Handbook of the Economics of Art and Culture, Elsevier North Holland, pp. 866-892.

Towse R. (2001), "Partly for the Money: Reward and Incentives to Artists", Kyklos, 54 2/3, pp. 473-490.

Wijnberg N.M., Gemser G. (2000), “Adding Value to Innovation: Impressionism and the Transformation of the Selection System in the Visual Arts", Organization Science, 11 (3), pp. 323-329.

\title{
Résumé
} La relation formation/carrières artistiques : le paradoxe des mondes de l'art

\author{
Nathalie Moureau et Dominique Sagot-Duvauroux
}

L'histoire de l'art, depuis le début du XXe siècle, s'est employée à dissocier le statut d'artiste de la maîtrise d'un savoir faire plastique (dessin, peinture, sculpture, etc.) en valorisant avant tout l'originalité de la démarche; pourtant, les écoles des beaux arts apparaissent encore aujourd'hui comme un passage quasi incontournable pour une carrière artistique sur le marché de l'art contemporain. Symétriquement, alors que la maitrise d'un savoir faire artisanal apparaît comme le principal critère d'appréciation de la qualité dans le monde de l'art classique s'inscrivant dans la tradition, il n'est pas rare que ces artistes soient autodidactes. Cet article explique ce paradoxe en mettant à jour les liens qui unissent les conventions artistiques, les modalités d'accès à la notoriété et la formation. À cet effet, il s'appuie sur une enquête réalisée auprès de 134 acteurs de l'art actuel et 69 artistes plasticiens.

\section{Mots clés}

Métiers des arts plastiques, relation formation-emploi, savoir faire, enquête Journal of Economic Literature: J 44, J 23 